\title{
Numerical Calculations on Flow and Heat Transfer in Smooth and Ribbed Two-Pass Square Channels under Rotational Effects
}

\author{
Zhongyang Shen, ${ }^{1}$ Yonghui Xie, ${ }^{1}$ Di Zhang, ${ }^{2}$ and Gongnan Xie ${ }^{3}$ \\ ${ }^{1}$ School of Energy and Power Engineering, Xian Jiaotong University, Xian, Shaanxi 710049, China \\ ${ }^{2}$ Key Laboratory of Thermo-Fluid Science and Engineering, Ministry of Education, School of Energy and Power Engineering, \\ Xian Jiaotong University, Xian, Shaanxi 710049, China \\ ${ }^{3}$ School of Mechanical Engineering, Northwestern Polytechnical University, P.O. Box 552, Xian, Shaanxi 710072, China
}

Correspondence should be addressed to Yonghui Xie; yhxie@mail.xjtu.edu.cn

Received 30 March 2014; Accepted 5 May 2014; Published 15 May 2014

Academic Editor: Haochun Zhang

Copyright (C) 2014 Zhongyang Shen et al. This is an open access article distributed under the Creative Commons Attribution License, which permits unrestricted use, distribution, and reproduction in any medium, provided the original work is properly cited.

\begin{abstract}
U-shaped channel, which is also called two-pass channel, commonly exists in gas turbine internal coolant passages. Ribbed walls are frequently adopted in internal passage to enhance the heat transfer. Considering the rotational condition of gas turbine blade on operation, the effect of rotation is also investigated for the coolant channel which is close to real operation condition. Thus, the objective of this study is to discuss the effect of rotation on fluid flow and heat transfer performance of U-shaped channel with ribbed walls under high rotational numbers. Investigated Reynolds number is $\mathrm{Re}=12500$ and the rotation numbers are $\mathrm{Ro}_{b}=0.4$ and 0.6. In the results, the spatially heat transfer coefficient distributions are exhibited to discuss the effect of rotation and roughened walls. It is found that ribbed walls enhance the heat transfer rate significantly. Under the rotational condition, the $\mathrm{Nu}$ in the first pass with outward flow is increased while that in the second pass is decreased. Finally, average Nu ratio, friction ratio, and thermal performance are all presented to discuss the thermal characteristics.
\end{abstract}

\section{Introduction}

Gas turbine is one kind of widely used power machineries which has been applied in land use power generation system and aircraft propulsion. The continuous development of gas turbine requires more and more efficient cooling technology for turbine blade. Han [1] conducted a lot of work in this area and demonstrated a schematic diagram of typical techniques for turbine blade cooling process which is presented in Figure 1. Among these techniques, rib turbulated cooling in internal passage is the most commonly adopted approach in gas turbine blade. Han and his coworkers [2-8] have conducted a large amount of research in this area including the discussions of aspect ratio, Reynolds number, channel shape, rib configurations, and so on.

In gas turbine blade, serpentine channel which is also called U-shaped channel with a 180 degree sharp bend is more common and complex than straight cooling channel as is discussed by Ekkad et al. [9] who considered the effect of secondary flow induced by ribs and 180 degree bend. Walker and Zausner [10] used commercial solver ANSYS CFX to predict heat transfer in a ribbed two-pass channel and indicated that $k-w$ SST is an optimal turbulence model for engineering applications. Bonhoff et al. [11] numerically investigated a two-pass channel with 45 degree ribs using the commercial solver FLUENT with Reynolds stress model (RSM) and standard k- $\varepsilon$ model. Viswanathan and Tafti [12] conducted numerical simulation on a two-pass channel with ribs using the advanced LES (large eddy simulation) and DES (detached eddy simulation) to present the detailed flow characteristics.

The rotational effect in turbine blade affects the flow and heat transfer characteristics considerably. Rotation induces Coriolis and centrifugal forces that produce cross-stream secondary flow in the rotating coolant passages. Especially in U-shaped channel, the effect of rotation on the first pass and second pass is different due to the different outward flow and inward flow. Agarwal et al. [13] investigated the heat transfer in 1:4 rectangular passages with rotation. They performed experimental test on smooth and ribbed walls using the naphthalene sublimation technique. The influence 


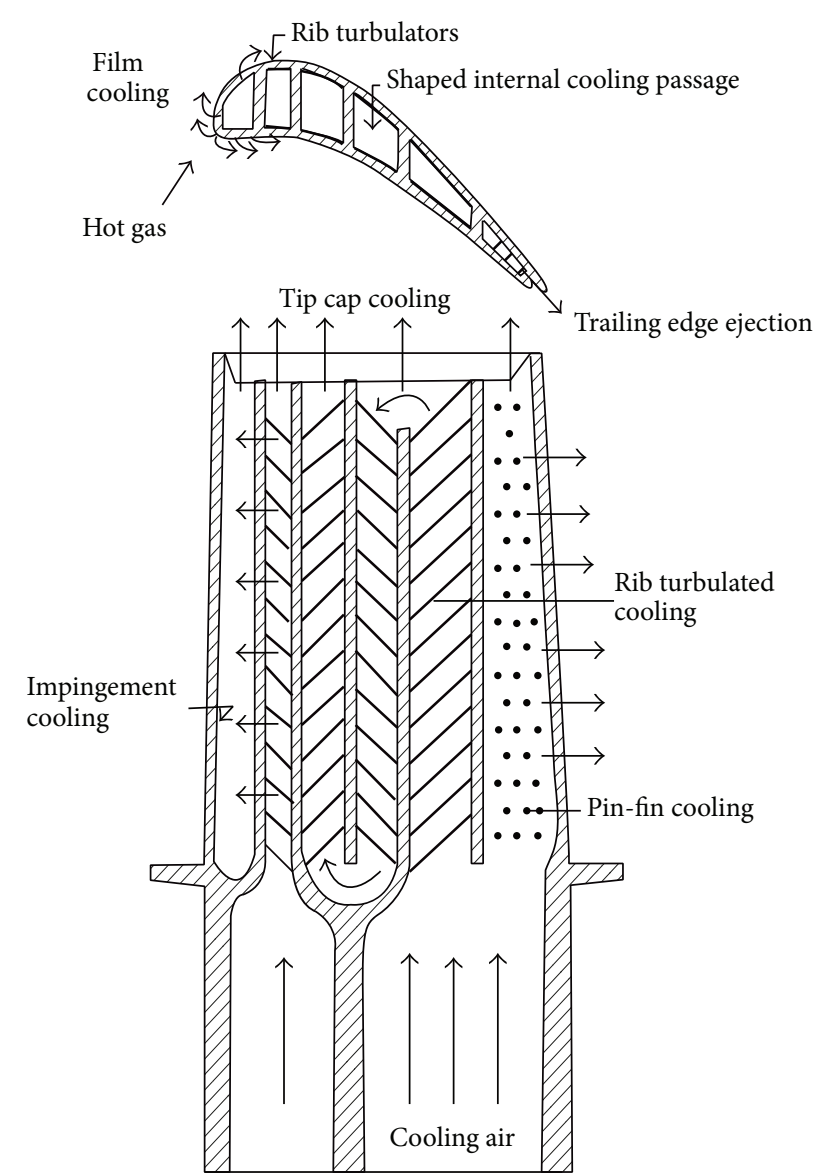

Figure 1: A schematic diagram of typical techniques for turbine blade cooling process [1].

of channel orientation on heat transfer in two-pass channel with smooth and ribbed walls when $\mathrm{AR}=2: 1$ is conducted by Huh et al. [14] whose main focus is the rotational orientation. Murata and Mochizuki [15] adopted large eddy simulation to investigate the turbulent heat transfer performance for a ribbed channel with turning bend. Liu et al. [16] investigated the effect of rib spacing which is varied in a large range on heat transfer in rotating two-pass channel $(\mathrm{AR}=1: 2)$. They compared the heat transfer characteristics in different rotational number with different rib space. In order to be similar with real blade coolant passage, a modified entrance is added to the channel inlet as in the literature of Huh et al. [17] and Schüler et al. [18]. In their study, different entrance modifications were made to make the fluid flow close to real engine conditions. They concluded that the effect of entrance inlet on the heat transfer performance cannot be ignored. The effect of entrance was also documented by Bunker [19] considering the entropy generation. Other attempts were also made to measure the heat and mass transfer of ribbed rectangular channel on bottom heated surface by Galanis and Rashidi [20]. To the author's knowledge, the investigation of the effect of high rotational number is not as abundant as rotational number, which is up to 0.2 for two-pass channel due to the experimental restrictions. Also,

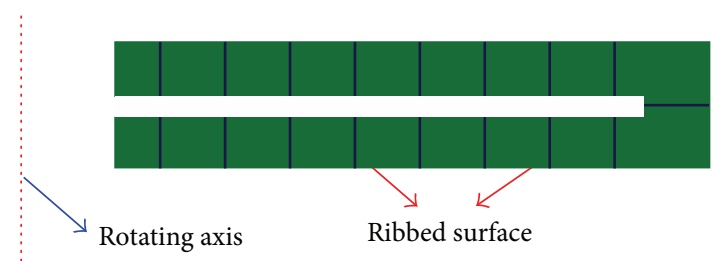

FIGURE 2: Schematic diagram of U-shaped channel with ribbed walls.

most of studies on the fluid flow and heat transfer in twopass channel are based on experimental tests. The detailed flow characteristics, especially the friction penalty, are not referred to in the literature. Thus, we conducted numerical predictions on the fluid flow and heat transfer performance on a two-pass channel under a high rotational number of up to 0.6 and presented the flow performance and friction factor to reveal the overall thermal performance. Results which are presented include surface Nusselt number distributions, channel friction factors, and overall thermal performance parameters under different conditions.

\section{Description of the Problem}

Numerical calculations were conducted on U-shaped cooling channel with ribbed walls. The baseline smooth U-shaped channel is referred to from the test model conducted by Tariq et al. [21]. Figure 2 is a schematic diagram of the test channel. As shown in this figure, roughened ribs are allocated in the first and second pass of channel. An additional rib is also placed at the center of the turning bend in order to enhance the heat transfer again in this area.

Both inlet and outlet of the channel are composed by a rectangular cross-section with a channel aspect ratio $(W / H)$ of $1: 1$. The hydraulic diameter of the inlet cross-section is $69.9 \mathrm{~mm}$ according to the value of $W$ and $H$. Typical parameters of U-shaped channel are length of single pass way $L 1=800 \mathrm{~mm}$, the flow gap of the 180 degree bend $L 2=$ $88.9 \mathrm{~mm}$, and tip distance of the turning bend $L 3=25.4 \mathrm{~mm}$. An extended adiabatic inlet segment with a length of 15 times the hydraulic diameter was added in order to obtain a relative fully developed flow. Arrays of square ribs $(a / e=1)$ in an inline arrangement are arranged on one surface of the channel which is clearly exhibited in Figure 2 while other surfaces are all smooth. The rib block ratio $\left(e / D_{h}\right)$ is 0.065 and the height normalized pitch $\left(P_{r} / D_{h}\right)$ is 1.25 . The placement of the ribs is orthogonal to the flow direction.

Since rotational conditions are discussed in this study, the location of the rotation axis and rotational orientation are worth mentioning. Figure 2 also presents coordinate system and information about rotation from two coordinate directions. The rotational direction is identified by stating that ribbed surface is the rotating trailing surface.

For the mesh generation, all meshes on ribbed surface and turning bend regions are made quite denser to successfully capture the heat transfer performance which results in the fact that $y+$ is near $0.5-1$ at all heated surfaces. After the grid independency study, the final adopted mesh numbers for 
smooth and ribbed channels are approximately 0.8 and 2.6 million.

For the computational study, Navier-Stokes equations were solved using the commercial finite-volume solver CFX with the turbulence model of $k$-w-SST model. The solutions of the conservation equation of mass, momentum, energy, and turbulence transport were converged with high resolution scheme. The rotation was realized by employing body force terms in the momentum and energy equation. In this paper, the channel inlet Reynolds number of 12500 was considered. The flow at the inlet owns a constant temperature of $300 \mathrm{~K}$ and a turbulent intensity of $5 \%$. The outlet of the domain in the second channel part is of stable average pressure of $1 \mathrm{~atm}$. Thermal boundary was imposed on the ribbed wall with constant heat flux ratio. For rotational cases, rotation factors were considered with values of 0.0, 0.4, and 0.6.

The Reynolds number in the present study is defined by

$$
\operatorname{Re}=\frac{\rho V_{m, \text { in }} D_{h}}{\mu} .
$$

The Nusselt number $\mathrm{Nu}$ is described by the following two equations:

$$
\begin{gathered}
\mathrm{Nu}=\frac{h D_{h}}{\lambda}, \\
h=\frac{q^{\prime \prime}}{T_{w}-T_{f}},
\end{gathered}
$$

where $h$ is the heat transfer coefficient and $\lambda$ is the thermal conductivity of air based on the mean temperature of the inlet and outlet temperatures. $T_{w}$ is the local wall temperature distribution. $T_{f}$ is the mass-weighted average temperature between the inlet and outlet of corresponding segments including first pass, turning bend, and second pass. Baseline Nusselt number is obtained by

$$
\mathrm{Nu}_{0}=0.023 \mathrm{Re}^{0.8} P_{r}^{0.4} .
$$

The average friction coefficient $f$ is described by the following equation:

$$
f=\frac{\Delta P}{4\left(\Delta L / D_{h}\right)(1 / 2) \rho V_{m, \text { in }}^{2}},
$$

where $\Delta P$ is the pressure drop between the channel inlet and outlet sections and $\Delta L$ is the streamwise length the fluids pass through. Baseline friction ratio $f_{0}$ is proposed by Petukhov using the Karman-Nikurade equation which is obtained by

$$
f_{0}=2(2.236 \ln \operatorname{Re}-4.639)^{-2} .
$$

\section{Results and Discussions}

Figure 3 presents the limiting streamlines on the surface and three-dimensional streamlines near the bend for the smooth channel. No excursion is observed until the fluids pass the sharp turning bend. Bend induced dean vortices are obviously observed in this region. Due to the excursion of fluids through the bend, centrifugal force drives the fluids towards the inner surface as the arrow shows in Figure 3(a). From the 3D streamlines near the bend shown in Figure 3(c), severe turbulence and secondary flow patterns exist in this area. It is also worth pointing out that the $3 \mathrm{D}$ streamlines are colored by turbulence kinetic energy. The TKE of fluids after the bend is more considerable than that in the first pass and this is also a reason for the difference of heat transfer performance in the two channel passes. In Figure 3(b), streamlines for rotational condition (take $\mathrm{Ro}_{b}=0.6$ as an example) are shown. Similar flow patterns exist for the rotation condition. However, the deviations of flow streamlines in the second pass differ from those in the stationary case.

For ribbed channel, other secondary flow patterns can be observed near the rib surface. Figures 4(a) and 4(c) plot the limiting streamlines near the ribbed bend region and corresponding $3 \mathrm{D}$ streamlines. It can be observed that rib surface disturbs the fluid flow through the channel. The impingement on rib front surface and separation area behind rib can be observed from the streamlines. The highly vortical fluids near the rib and bend can be seen from the 3D streamlines. The highly turbulent flow results in more energized fluid flow accompanied with high heat transfer rate.

The local heat transfer distributions of the smooth and ribbed two-pass configurations are shown in Figures 5 and 6 separately. The $\mathrm{Nu} / \mathrm{Nu}_{0}$ distributions on the heated surface for smooth channel are shown in Figure 6. It is observed that high heat transfer rate exists near the inlet region which decays towards the bend area. The Nu distribution is symmetric until the bend area which is in accordance with the flow patterns. Flow impingement and Dean vortices led to areas of considerably higher heat transfer on the tip wall and the outlet pass sidewall. Heat transfer in the outlet pass downstream of the bend was strongly enhanced because of local flow constriction due to the flow deviation and separation bubbles and it is rapidly decayed in about 3$4 d_{h}$ and approaches stable until the channel outlet. While with rotation, in the first pass, the fluids are shifted towards the trailing surface and hence enhance the heat transfer in these areas because the flow in the first pass is outward flow. The combined effect of rotating centrifugal force and bend induced secondary flow makes the heat transfer in bend region close to the stationary case. In the second pass, the rotating force for inward flow condition decreases the heat transfer in the trailing surface as is shown.

Figure 6 plots the $\mathrm{Nu} / \mathrm{Nu}_{0}$ distributions for the ribbed two-pass configuration. For the none-rotating condition, the heat transfer in the first pass is mainly dominated by ribinduced secondary flow. Typical $\mathrm{Nu}$ distribution pattern of square rib is observed from the detailed pictures. Nu ratio is higher near the inlet due to the entrance effect and decreases along the flow direction until the turning bend which is almost symmetric. The $\mathrm{Nu}$ value of the section most close to the bend is almost close to the baseline $\mathrm{Nu}_{0}$. Higher $\mathrm{Nu}$ occurs on the bend region than the smooth channel due to the rib-induced flow patterns especially the middle rib across the bend centerline. Coming to the second pass, the deviation of fluids by the bend and rib-induced vortex mix with each other. Similar heat transfer distributions can be 


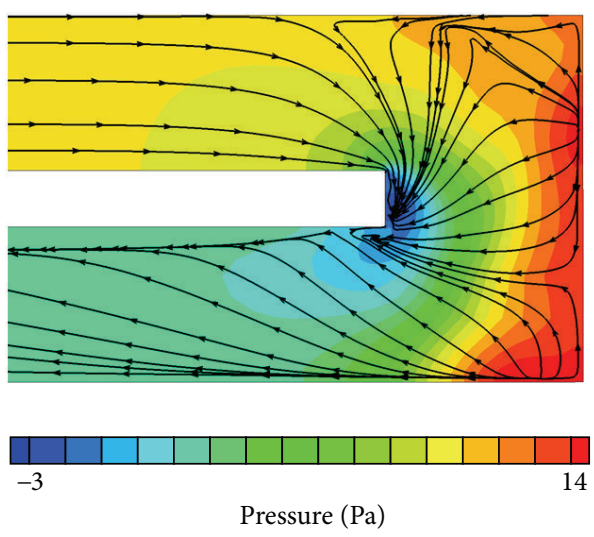

(a)

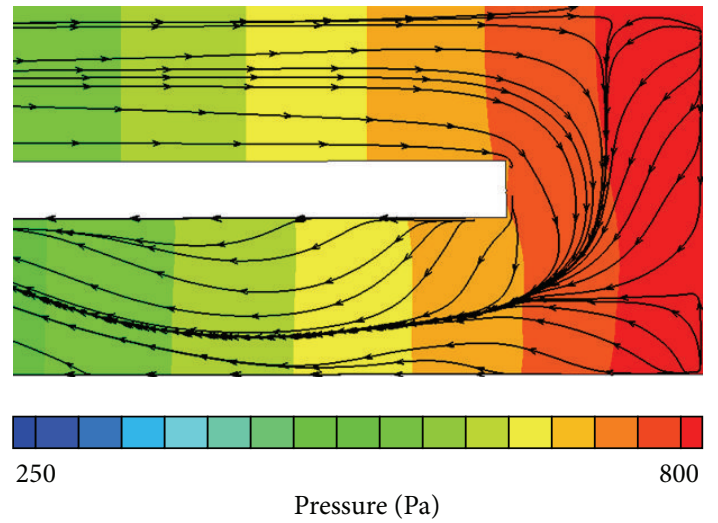

(b)

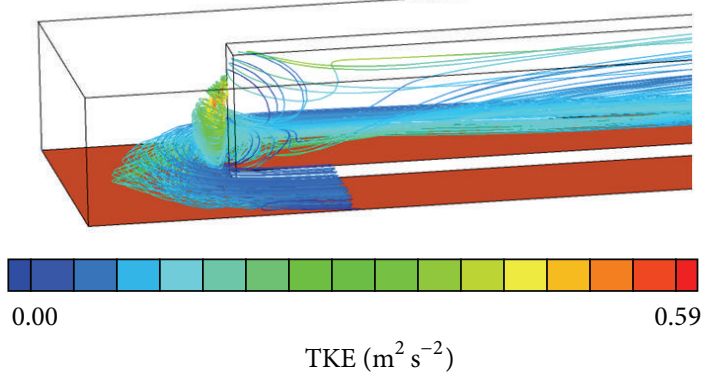

(c)

FIGURE 3: Streamlines for smooth U-shaped channel: (a) limiting streamlines $\left(\operatorname{Ro}_{b}=0\right)$, (b) limiting streamlines $\left(\operatorname{Ro}_{b}=0.6\right)$, and $(c) 3 D$ streamlines near the turning bend.

observed at the different sections, which decay gradually. Heat transfer distribution for rotational case is shown in Figure 6(b). Similar with the smooth channel, heat transfer coefficient is increased in the first pass and the distribution patterns between successive ribs are different from stationary case because the rotating Coriolis force makes the fluids shift and impinge on the trailing surface. $\mathrm{Nu}$ is obviously enhanced in the first pass but the values on the bend region are even slightly decreased. In the second pass, rotation Coriolis force interacts with the rib-induced vortex which decreases the heat transfer rate. The similar distribution pattern of sections between ribs disappears which is also exhibited in the streamlines above where fluid streamlines deviate between outer sidewall and the divider. Above all, the effect of rotation on the second pass is more marginal than other areas.

Tables 1 and 2 show the averaged heat transfer enhancement ratio and friction penalty. It is concluded that, for smooth channel, the increase of rotational number leads to lower averaged heat transfer rate. While for ribbed channel, due to the change of flow separation zone, the averaged heat transfer rate is higher at higher rotational number. For all channels, higher friction ratio is obtained at higher rotational number due to larger wall shear.

\section{Conclusions}

In the present study, numerical researches were conducted on the heat transfer performance in U-shaped channel arranged
TABLE 1: Averaged $\mathrm{Nu} / \mathrm{Nu}_{0}$ for all channels under different conditions.

\begin{tabular}{lccc}
\hline $\mathrm{Nu} / \mathrm{Nu}_{0}$ & $\mathrm{Ro}_{b}=0.0$ & $\mathrm{Ro}_{b}=0.4$ & $\mathrm{Ro}_{b}=0.6$ \\
\hline Smooth channel & 1.602 & 1.586 & 1.585 \\
Ribbed channel & 1.745 & 1.883 & 1.976 \\
\hline
\end{tabular}

TABLE 2: Averaged $f / f_{0}$ for all channels under different conditions.

\begin{tabular}{lccc}
\hline$f / f_{0}$ & $\mathrm{Ro}_{b}=0.0$ & $\mathrm{Ro}_{b}=0.4$ & $\mathrm{Ro}_{b}=0.6$ \\
\hline Smooth channel & 3.397 & 3.541 & 4.257 \\
Ribbed channel & 4.273 & 4.297 & 4.815 \\
\hline
\end{tabular}

with ribbed walls. The objective of this study is to discuss the combined effect of rib and rotation on thermal performance of U-shaped channel. Specific conclusions are as follows.

In the U-shaped channel, heat transfer rates in secondary pass are obviously higher than those in the first pass. Complex flow separation, reattachment, and deflection with significant secondary flow phenomenon exist near the 180 degree turning bend and result in high heat transfer rates in this area. Rib structure will enhance the heat transfer rate due to flow constriction and reattachment.

Under the effect of rotation, the Coriolis force will increase the heat transfer rate in the first pass for outward flow while it will decrease the heat transfer rate in the second pass as inward flow. For the averaged $\mathrm{Nu}$ of the channel, 


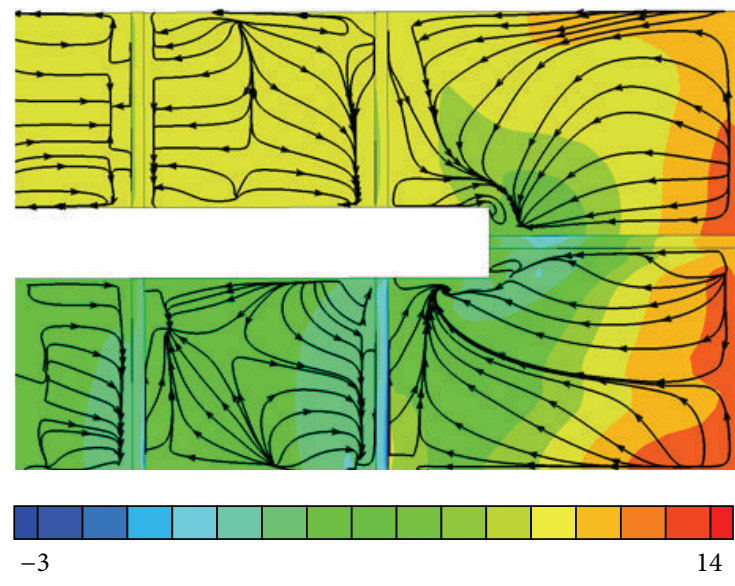

Pressure $(\mathrm{Pa})$

(a)

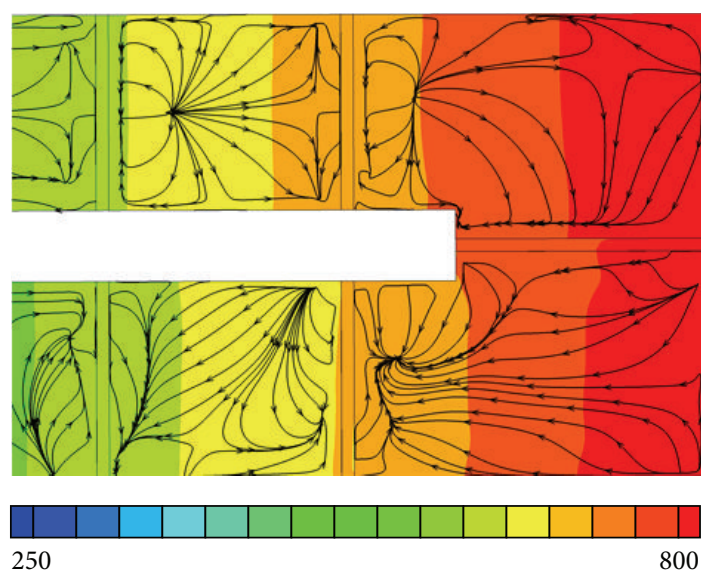

Pressure $(\mathrm{Pa})$

(b)

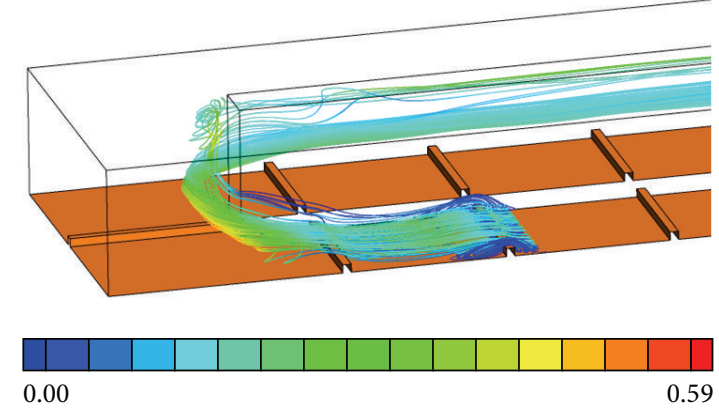

$\operatorname{TKE}\left(\mathrm{m}^{2} \mathrm{~s}^{-2}\right)$

(c)

FIGURE 4: Streamlines for ribbed U-shaped channel: (a) limiting streamlines $\left(\operatorname{Ro}_{b}=0\right)$, (b) limiting streamlines $\left(\mathrm{Ro}_{b}=0.6\right)$, and $(\mathrm{c}) 3 \mathrm{D}$ streamlines near the turning bend.

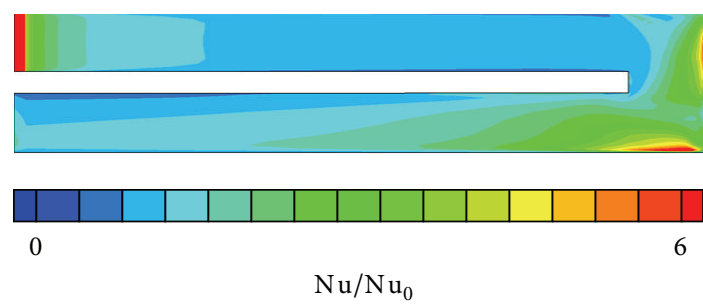

(a)

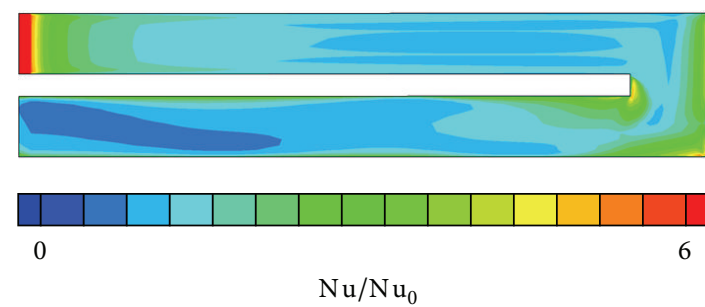

(b)

FIGURE 5: $\mathrm{Nu} / \mathrm{Nu}_{0}$ of the smooth channel: (a) none-rotating case $\left(\mathrm{Ro}_{b}=0\right)$ and (b) rotating case $\left(\operatorname{Ro}_{b}=0.6\right)$.
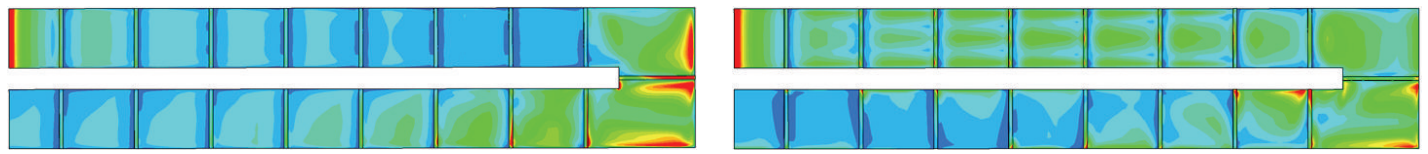

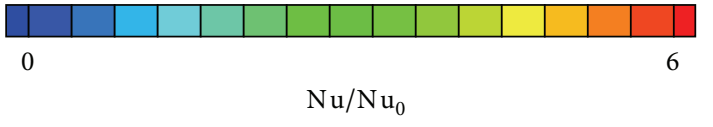

(a)

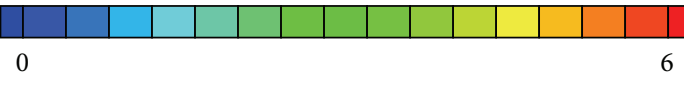

$\mathrm{Nu} / \mathrm{Nu}_{0}$

(b)

FIGURE 6: $\mathrm{Nu} / \mathrm{Nu}_{0}$ of the ribbed channel: (a) none-rotating case $\left(\mathrm{Ro}_{b}=0\right)$ and $(\mathrm{b})$ rotating case $\left(\operatorname{Ro}_{b}=0.6\right)$. 
the increase of rotational number leads to lower averaged heat transfer rate for smooth channel and higher heat transfer rate for ribbed channel. Larger friction loss is obtained under higher rotational number.

\section{Nomenclatures}

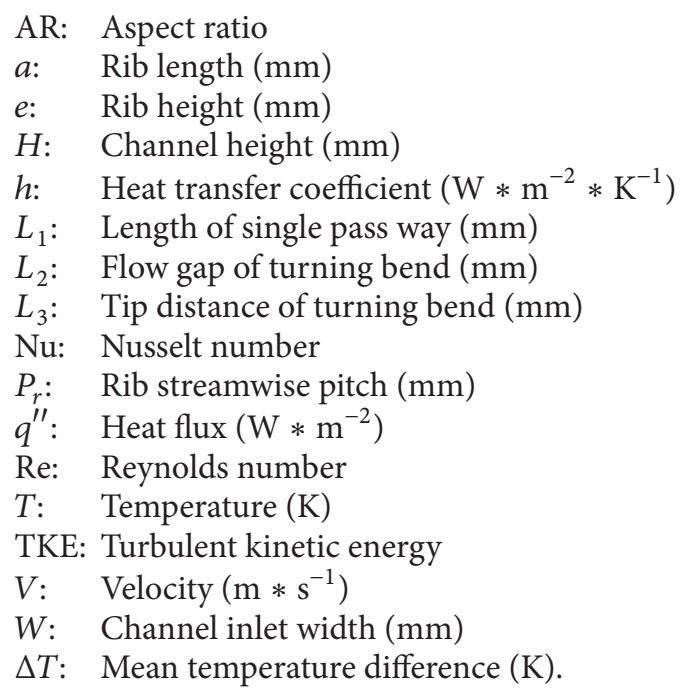

\section{Greek Symbols}

$$
\begin{aligned}
& \lambda: \text { Thermal conductivity }\left(W * \mathrm{~m}^{-1} * \mathrm{~K}^{-1}\right) \\
& \rho \text { : Density }\left(\mathrm{k} * \mathrm{~m}^{-3}\right) \text {. }
\end{aligned}
$$

\section{Subscripts}

in: Inlet

$\mathrm{m}$ : Mass flow average

out: Outlet

w: Wall.

\section{Conflict of Interests}

The authors declare that there is no conflict of interests regarding the publication of this paper.

\section{References}

[1] J.-C. Han, "Turbine blade cooling studies at Texas A\&M University: 1980-2004," Journal of Thermophysics and Heat Transfer, vol. 20, no. 2, pp. 161-187, 2006.

[2] J. C. Han, L. R. Glicksman, and W. M. Rohsenow, "An investigation of heat transfer and friction for rib-roughened surfaces," International Journal of Heat and Mass Transfer, vol. 21, no. 8, pp. 1143-1156, 1978.

[3] J. C. Han, "Heat transfer and friction in channels with two opposite rib roughened walls," Journal of Heat Transfer, vol. 106, no. 4, pp. 774-781, 1984.

[4] J. C. Han, "Heat transfer and friction characteristics in rectangular channels with rib turbulators," Journal of Heat Transfer, vol. 110 , no. 2, pp. 321-328, 1988.
[5] J. C. Han and J. S. Park, "Developing heat transfer in rectangular channels with rib turbulators," International Journal of Heat and Mass Transfer, vol. 31, no. 1, pp. 183-195, 1988.

[6] J. S. Park, J. C. Han, Y. Huang, S. Ou, and R. J. Boyle, "Heat transfer performance comparisons of five different rectangular channels with parallel angled ribs," International Journal of Heat and Mass Transfer, vol. 35, no. 11, pp. 2891-2903, 1992.

[7] G. S. Azad, M. J. Uddin, J.-C. Han, H.-K. Moon, and B. Glezer, "Heat transfer in a two-pass rectangular rotating channel with 45-deg angleb rib turbulators," Journal of Turbomachinery, vol. 124, no. 2, pp. 251-259, 2002.

[8] M. Huh, Y.-H. Liu, and J.-C. Han, "Effect of rib height on heat transfer in a two pass rectangular channel $(A R=1: 4)$ with a sharp entrance at high rotation numbers," International Journal of Heat and Mass Transfer, vol. 52, no. 19-20, pp. 4635-4649, 2009.

[9] S. V. Ekkad, Y. Huang, and J.-C. Han, "Detailed heat transfer distributions in two-pass square channels with rib turbulators and bleed holes," International Journal of Heat and Mass Transfer, vol. 41, no. 23, pp. 3781-3791, 1998.

[10] D. Walker and J. Zausner, "RANS evaluations of internal cooling passage geometries: ribbed passage and a 180 degree bend," ASME Paper GT2007-278, 2007.

[11] B. Bonhoff, U. Tomm, and B. V. Johnson, "Heat transfer predictions for U-shaped coolant channels with skewed ribs and with smooth walls," ASME Paper 96-TA-7, 1996.

[12] A. K. Viswanathan and D. K. Tafti, "Detached eddy simulation of turbulent flow and heat transfer in a ribbed duct," Journal of Fluids Engineering, vol. 127, no. 5, pp. 888-896, 2004.

[13] P. Agarwal, S. Acharya, and D. E. Nikitopoulos, "Heat transfer in 1:4 rectangular passages with rotation," Journal of Turbomachinery, vol. 125, no. 4, pp. 726-733, 2003.

[14] M. Huh, J. Lei, Y.-H. Liu, and J.-C. Han, "High rotation number effects on heat transfer in a rectangular $(A R=2: 1)$ two-pass channel," Journal of Turbomachinery, vol. 133, no. 2, Article ID 021001, 2010.

[15] A. Murata and S. Mochizuki, "Effect of rib orientation and channel rotation on turbulent heat transfer in a two-pass square channel with sharp $180^{\circ}$ turns investigated by using large eddy simulation," International Journal of Heat and Mass Transfer, vol. 47, no. 12-13, pp. 2599-2618, 2004.

[16] Y.-H. Liu, L. M. Wright, W.-L. Fu, and J.-C. Han, "Rib spacing effect on heat transfer in rotating two-pass ribbed channel ( $A R$ = 1:2)," Journal of Thermophysics and Heat Transfer, vol. 21, no. 3, pp. 582-595, 2007.

[17] M. Huh, J. Lei, and J.-C. Han, "Influence of channel orientation on heat transfer in a two-pass smooth and ribbed rectangular channel $(A R=2: 1)$ under large rotation numbers," Journal of Turbomachinery, vol. 134, no. 1, Article ID 011022, 2011.

[18] M. Schüler, H.-M. Dreher, S. O. Neumann, B. Weigand, and M. Elfert, "Numerical predictions of the effect of rotation on fluid flow and heat transfer in an engine-similar two-pass internal cooling channel with smooth and ribbed walls," Journal of Turbomachinery, vol. 134, no. 2, Article ID 021021, 2011.

[19] R. S. Bunker, "The augmentation of internal blade tip-cap cooling by arrays of shaped pins," Journal of Turbomachinery, vol. 130, no. 4, Article ID 041007, 2008. 
[20] N. Galanis and M. M. Rashidi, "Entropy generation in nonNewtonian fluids due to in the entrance region of ducts," Heat and Mass Transfer, vol. 48, no. 9, pp. 1647-1662, 2012.

[21] A. Tariq, K. Singh, and P. K. Panigrahi, "Detailed measurement of heat transfer and flow characteristics in rectangular duct with rib turbulators mounted on the bottom surface," Engineering Turbulence Modelling and Experiments, no. 5, pp. 445-454, 2002. 


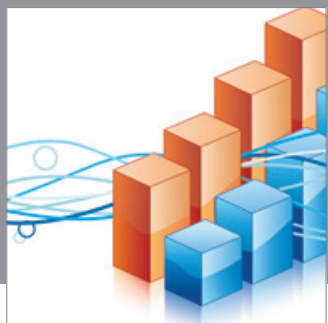

Advances in

Operations Research

mansans

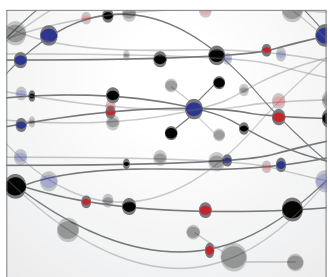

The Scientific World Journal
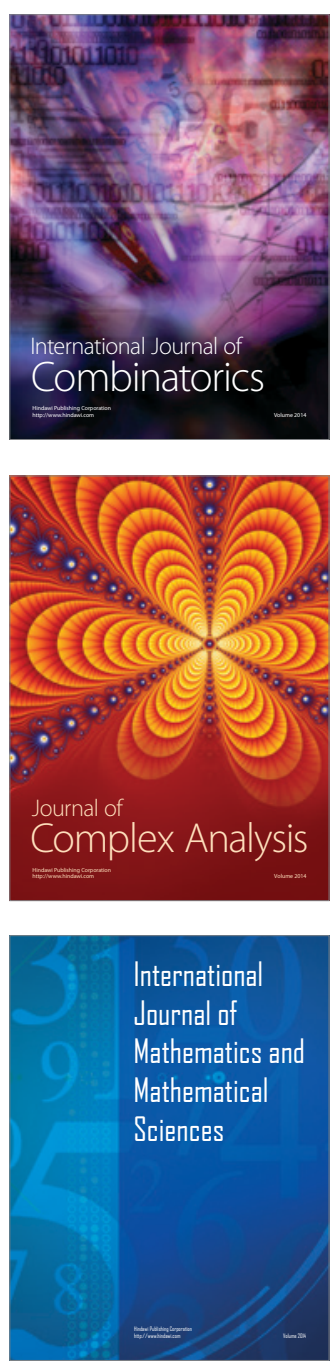
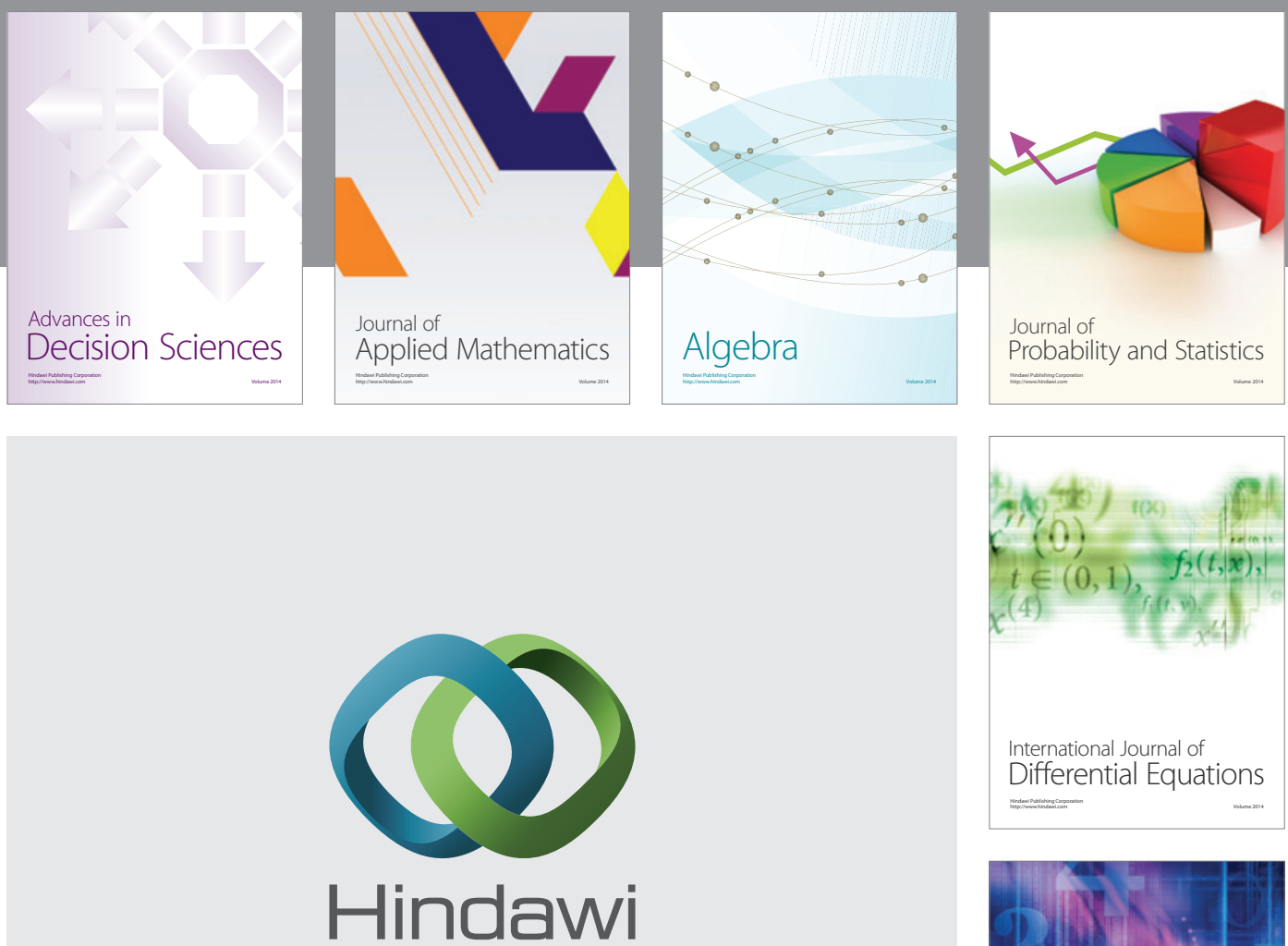

Submit your manuscripts at http://www.hindawi.com
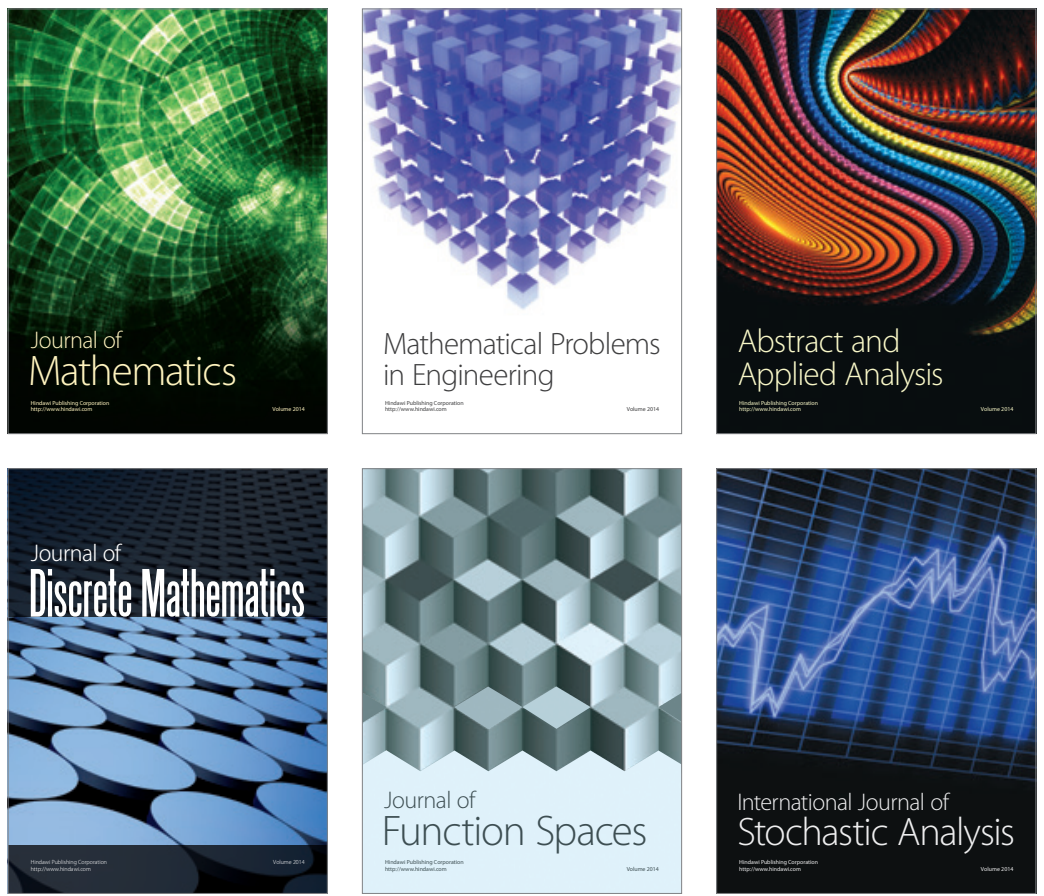

Journal of

Function Spaces

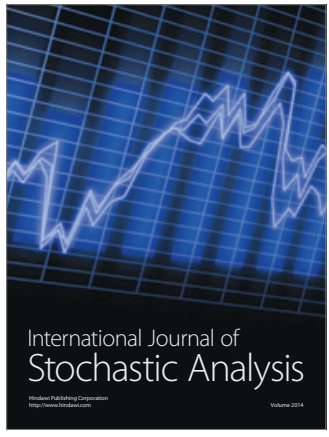

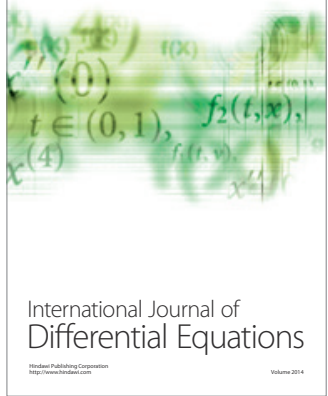
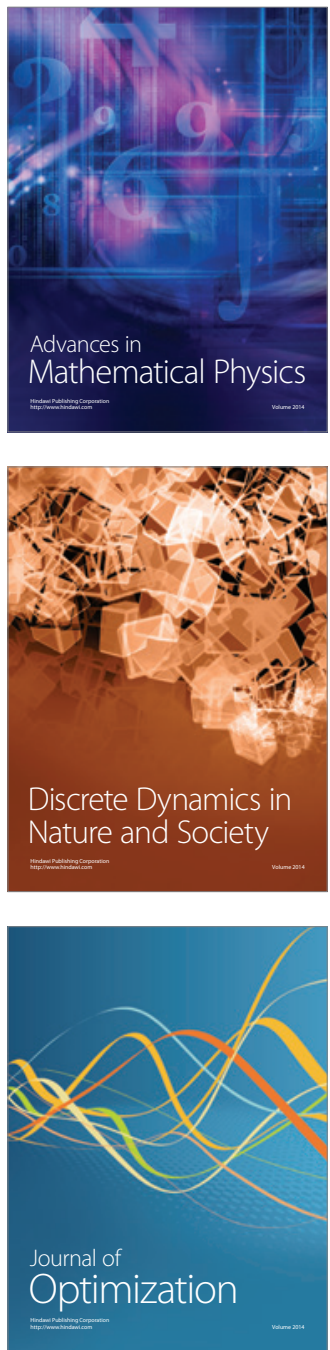